\title{
Growth kinetics of body centered cubic colloidal crystals
}

\author{
M. Würth, J. Schwarz, F. Culis, P. Leiderer, and T. Palberg* \\ Universität Konstanz, Postfach 5560 M676 D-78434 Konstanz, Germany
}

(Received 3 March 1995)

\begin{abstract}
A combination of static light scattering and video microscopy is used to perform high precision measurements on the growth velocity of body centered cubic (bcc) crystals in a metastable colloidal melt of monodisperse, highly charged latex spheres. The crystals nucleate heterogeneously at the walls of a flat flow-through shear cell and solidification proceeds without significant disturbance by homogeneous nucleation. The suspension parameters packing fraction $\Phi$ of the spheres and the concentration of screening electrolyte $c$ are systematically varied for two kinds of particles with equal diameter but different charge. For all experimental conditions the growth velocities in the $\langle 110\rangle$ direction collapse on a single curve if plotted against a reduced energy density difference $\Pi^{*}$ between the melt and the fluid at melting. Close to the phase boundary growth velocities vary linearly with increasing $\Pi^{*}$, and saturate at large $\Pi^{*}$ at a value of $v_{\infty}=9.1 \mu \mathrm{m} \mathrm{s}^{-1}$. The master curve can be fitted excellently by a Wilson-Frenkel growth law which was suggested to hold for the solidification of highly charged systems. A comparison of coefficients allows for the derivation of a quantitative estimation procedure for the difference in chemical potential $\Delta \mu$ between melt and solid in terms of the thermal energy $k_{B} T: \Delta \mu=\Pi^{*} B$. The best value for the conversion factor $B$ is found to be $B=(6.7 \pm 0.1) k_{B} T$. In contrast to previous work on homogeneously nucleated crystals the growth velocity of the $\langle 110\rangle$ face is limited by the reactionlike kinetics of registering preordered layers formed within an interface of finite thickness. We suggest a unified description covering also the growth of the rough interfaces of other crystal faces.
\end{abstract}

PACS number(s): 82.70.Dd, 61.50.Cj, 64.70.Dv

\section{INTRODUCTION}

The solidification of undercooled melts has been used in the processing of materials for a very long time. Nevertheless, our understanding of this important first order phase transition is still far from being comprehensive. During the first part of this century a number of semiempirical theoretical descriptions for the kinetics of nucleation and growth have been developed, mainly by adapting models of the condensation of liquid drops from the vapor phase [1-3]. Their verification in atomic or molecular systems faces two major problems. In most cases direct observation is difficult due to the high rates of nucleation and high velocities of growth. In addition, the relevant length scales are accessible only on immense technical effort. Consequently, experimental data of both high temporal and spatial resolution are sparse. Quantitative comparisons to the above mentioned theories, on the other hand, are often hindered by poorly defined or changing experimental conditions. For example, solidification usually starts during an applied temperature quench and is accompanied by the release of large amounts of latent heat together with limited thermal diffusion. This often prevents the development of sufficiently stationary conditions.

Colloidal crystals and melts, on the other hand, may be prepared and observed under conditions which in principle come very close to those defined by the assumptions

\footnotetext{
${ }^{*}$ Corresponding author.
}

of the theories and simulations mentioned above. The particle spacing is on the order of the wavelength of visible light, and the presence of the viscous suspending medium shifts the relevant time scales to a range between milliseconds and hours. This facilitates observations with high temporal and spatial resolution both in real and in reciprocal space by complementary optical techniques $[4,5]$.

Second, colloidal solids may easily be shear molten [6], and the phase transition is quasi-isothermal due to the low particle density of some $10^{-12}-10^{-14} \mathrm{~cm}^{-3}$ and the presence of the suspending fluid acting as a large heat sink. This allows for the formation of sufficiently stationary states after quasi-instantaneous quenches.

Finally, the interaction between the dispersed particles can be precisely adjusted [7] and determined [8] experimentally, while it is also well described by theoretical expressions $[9,10]$. This leads to a detailed description of suspension structures and dynamics including complications of polydispersity and hydrodynamic interaction [11-14]. For hard sphere systems freezing and melting are located at packing fractions of $\Phi_{f}=0.494$ and $\Phi_{m}=0.545$, respectively [15]. Suspensions with electrostatic stabilization solidify at considerably smaller packing fractions depending on the surface charge and the amount of screening electrolyte $[11,16,17]$.

These colloid specific advantages have led to a strong interest in the solidification dynamics of colloidal crystals. A considerable number of papers exist both on the nucleation [18-20] and the growth from the metastable shear melt [18,21-28]. These have been compared [29] to the classical theories of nucleation and growth [1-3] and to recent computer simulations [30,31]. In general, 
growth was observed to be much less affected by changes in the suspension parameters than nucleation [27]. In hard sphere systems one observes a $\sqrt{t}$ growth at short times $t$ and Lifshitz-Sloyzov ripening at late stages $[25,26]$. The underlying mechanism was inferred to be the formation of a depletion zone around the crystallites. As a function of packing fraction the growth coefficients show a sharp maximum in the upper coexistence range and decrease to zero as the glass transition is approached $[26,32]$.

In a first systematic study on highly charged systems Aastuen et al. reported linear growth and a slow but monotoneous increase in the growth velocity $v$ as a function of the packing fraction $\Phi$ [18]. To interpret their data they suggested a Wilson-Frenkel growth behavior:

$$
v=v_{\infty}\left[1-\exp \left(\frac{\Delta \mu}{k_{B} T}\right)\right],
$$

where $k_{B} T$ is the thermal energy. The limiting velocity $v_{\infty}$ was assumed to be determined by the lateral diffusion of particles towards their target place in the crystal lattice using the free Stokes-Einstein diffusion coefficient. The chemical potential difference $\Delta \mu$ between the melt and the solid was approximated by the reduced difference in the particle densities $\rho$ at the actual experimental conditions and at melting. A fit of expression (1) to the data was consistent with the suggested growth law. However, a quantitative verification using samples of more precisely defined parameters and a well known phase diagram is still missing.

Very recently a study was performed on suspensions of weakly charged silica spheres [27]. Time resolved measurements of the static structure factor showed a linear increase of the integrated intensity of the (111) Bragg reflection. This intensity is proportional to the number of crystallites times their volume. The rate of intensity increase $R$ showed a pronounced maximum as a function of the packing fraction. Further evaluation using a model with cubic crystal geometry showed no significant variation of the increase in linear crystal dimension as a function of effective packing fraction. Clearly this is incompatible with Eq. (1). The nature of the velocity limiting process in charged systems therefore remains unresolved.

In this paper we report measurements of the growth velocity in suspensions of highly charged latex spheres spanning the whole range from the coexistence regime to large "undercooling." We focus on the propagation of a planar interface in a well defined crytallographic direction without interference by simultaneous nucleation processes. This facilitates the use of a high precision detection scheme for the position of the propagating interface as a function of time. The velocity of growth is directly inferred from the recorded video frames and no models and assumptions have to be made in its derivation.

Our investigation differs from previous ones since we use a recently reported advanced deionization technique [7] to prepare homogeneous and gradient-free metastable melts of precisely controlled suspension parameters. We systematically vary all relevant suspension parameters for highly charged systems: packing fraction, concentration of screening electrolyte, and surface charge. Our results unequivocally confirm a Wilson-Frenkel growth law for highly charged systems.

The second important difference is a qualitative different model of the microscopic structure of the interface. While a number of previous papers are concerned with the shape of the interface, much less is known about its structure. It has been assumed that the interface is microscopically sharp. This stands in contrast to the molecular dynamics simulations of a Lennard-Jones solid growing from the melt [30,31], Brownian dynamics simulations [33], and density functional theory calculations $[34,35]$ on the equilibrium shape of the solid-liquid interface at coexistence. These models indicate the possibility of a thick interface consisting of several layers of particles which show a continuous decay of crystalline order into the melt. We will use the latter concept in combination with the Wilson-Frenkel growth law. Inserting the long time self-diffusion coefficient instead of the StokesEinstein value gives the possibility to use a unified description of growth in systems without pronounced depletion zones.

Our paper is organized as follows: the preparation and detection techniques form the basis of quantitative measurements. Therefore they are presented in detail in the experimental section together with a characterization of the particles under study. The results on the growth velocity and their evaluation in terms of a reduced density difference is presented in Sec. III. Section IV contains a discussion on the structure of the interface and the range of applicability of Wilson-Frankel growth.

\section{SYSTEM AND EXPERIMENTAL METHODS}

We used two kinds of commercially available polystyrene latex spheres (Seradyn, Inc., U.S.A; Lot No. 2010 M9R and Lot No. 2011 M9R) denoted sample $A$ and $B$, both with hydrodynamic radius $a=51 \mathrm{~nm}$ (dynamic light scattering) and a titrated number of sulfate surface groups of $N_{A}=950$ and $N_{B}=1200$, respectively. The particles with the lower charge number (sample $A$ ) are identical to the ones used in previous studies $[36,8,10]$. Both particles have been used in the study of structure and dynamics of fluid mixtures [37]. Their data are summarized in Table $\mathbf{I}$.

The continuous deionization technique, which was described in detail elsewhere [7], allows the precise control of the experimental parameters with typical uncertainties in the packing fraction $\Phi$ and salt concentration $c$ below $2 \%$. This in turn facilitates the recording of an accurate $\Phi-c$ phase diagram. The respective phases are identified by microscopic and static light scattering methods. We show the phase diagram of the $A$ particles in Fig. 1. The fluid and the bcc crystalline phase are separated by a coexistence region which broadens with increasing $\Phi$ and $c$. Note the low values of packing fraction and salt concentration which significantly extend the previously studied range of experimental parameters of these particles [38]. The phase diagram of sample $B$ is not explicitly shown. It is of very similar shape but the freezing transition is shifted towards slightly lower values of $\Phi$ and 
TABLE I. The best parameters of the fits to each single series.

\begin{tabular}{cccccccc}
\hline \hline Sample & $a(\mathrm{~nm})$ & $\Phi$ & $c\left(\mu \mathrm{mol}^{-1}\right)$ & \multicolumn{1}{c}{$N$} & $Z_{\mathrm{PBC}}$ & $v_{\infty}\left(\mu \mathrm{m} \mathrm{s}^{-1}\right)$ & $B\left(\right.$ units of $\left.k_{B} T\right)$ \\
\hline$A$ & 51 & 0.003 & $0-1.0$ & 950 & 395 & $9.05 \pm 0.1$ & $6.69 \pm 0.1$ \\
$B$ & 51 & 0.0022 & $0-1.2$ & 1200 & 450 & $9.09 \pm 0.1$ & $6.66 \pm 0.1$ \\
$B$ & 51 & $0-0.003$ & 0 & 1200 & 450 & $9.13 \pm 0.1$ & $6.72 \pm 0.1$ \\
\hline \hline
\end{tabular}

higher values of $c$.

The present investigations on the crystal growth velocities cover packing fractions of $0.001 \leq \Phi \leq 0.003$ $\mu$ mol $c$, where $\Phi$ converts to the particle number density $\rho$ as $\rho=\Phi / V_{P}$ with the particle volume $V_{P}$. Under these conditions crystals nucleate heterogeneously at the wall and grow in an undisturbed way. At larger $\Phi$ homogeneous nucleation becomes the predominant mechanism of solidification and interferes with wall crystal growth.

In preliminary experiments we observed that large monolithic crystals filling the whole measuring cell could be grown if shear melting was performed under conditions of constant flow. We therefore modified the preparational setup reported in [7]. The suspension flows through a $1 \times 10 \mathrm{~mm}^{2}$ rectangular optical cell (Rank, Bottisham, UK) of $40 \mathrm{~mm}$ length. Both ends of the cell are connected to reservoirs to apply a constant hydrostatic pressure difference. The higher reservoir is refilled by a peristaltic pump. The flow profile throughout the cell is parabolic and constant in time. Care was taken to avoid convection after stopping the flow by using electromagnetic values. The suspension is left in a mechanically undisturbed shear molten state which has recently been characterized as a metastable melt by measurements of its diffusional properties [36].

Crystal growth starts immediately at the cell walls and is monitored with the setup sketched in Fig. 2(a). The cell is observed side on by a long distance telescopic microscope (QM 100, Questar NL). A laser beam crosses the cell under an angle $\Theta$ with respect to the observation

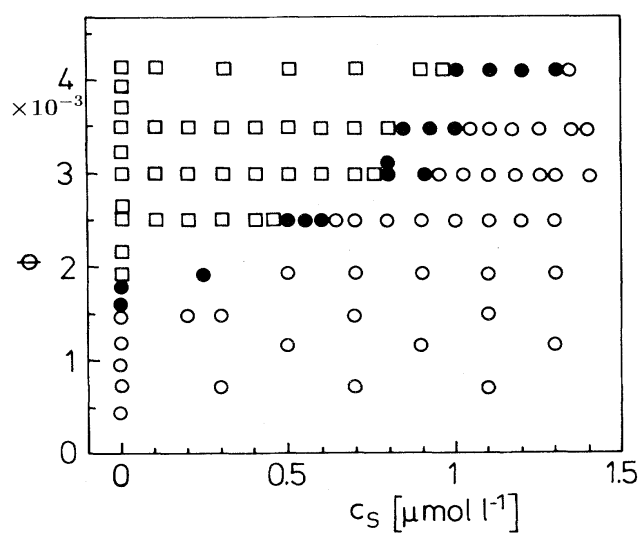

FIG. 1. The experimental phase diagram of the $A$ particles in dependence on the salt concentration $c$ and the volume fraction $\Phi$. Squares denote the bcc phase and open circles the fluid phase. The two phases are divided by a coexistent region denoted by closed circles. direction. This corresponds to a scattering vector of magnitude $q=(4 \pi n / \lambda) \sin (\theta / 2)$ for which the intensity of scattered light largely differs for the fluid and the solid phases, as is schematically shown in Fig. 2(b). The image of the beam is recorded by a charge-coupled-device (CCD) camera and further evaluated by image processing (OFG, Stemmer, FRG).

In Figs. 3(a)-3(d) we show four representative snapshots of the laser beam crossing the sample cell. In Fig. 3(a), the sample is still sheared and the image appears as a broad light band. Upon stopping the flow single speckels are discernible. The third picture is taken 60 $s$ after the start of solidification; the intensity difference between the two phases is clearly visible. Finally, after complete solidification, the cell is filled with two monolithic wall crystals joined in the cell center. Again, inten-
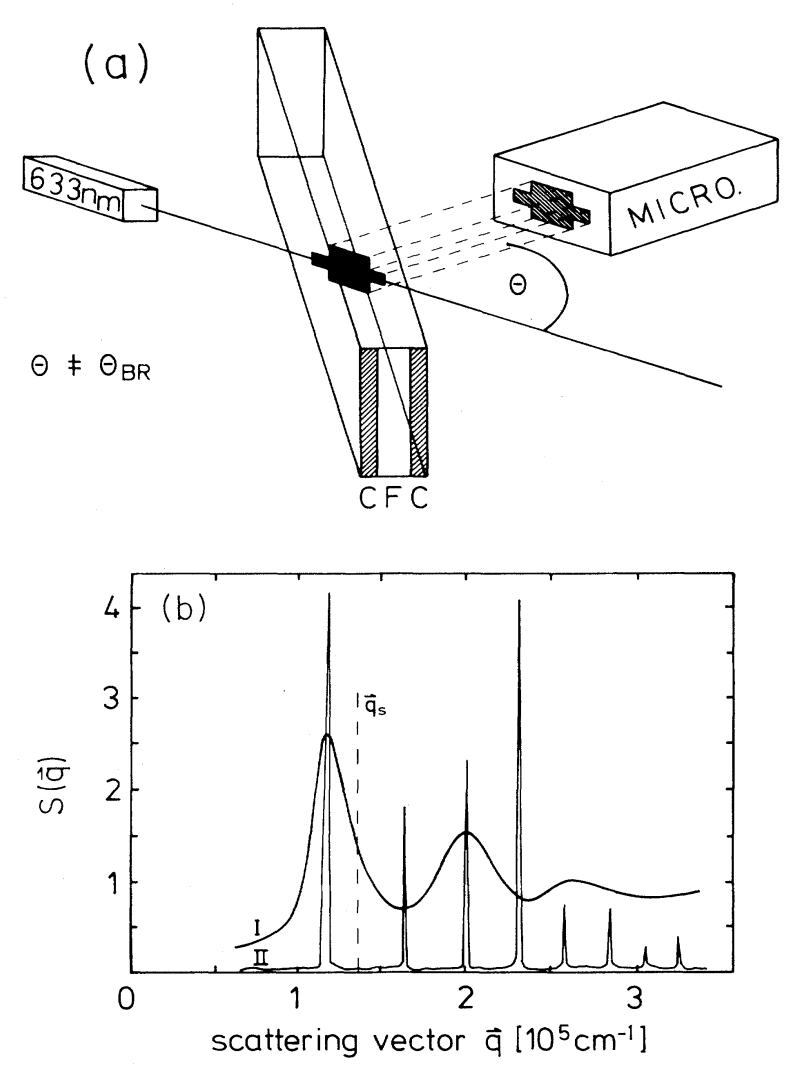

FIG. 2. (a) Optical setup used for measuring the growth velocity. $C$ denotes the crystalline phase growing against the metastable melt $F$. (b) The scattered intensities for fluid (I) and solid (II) phase. Note their large difference at $q_{s}$, which is indicated by vertical dashed line. 


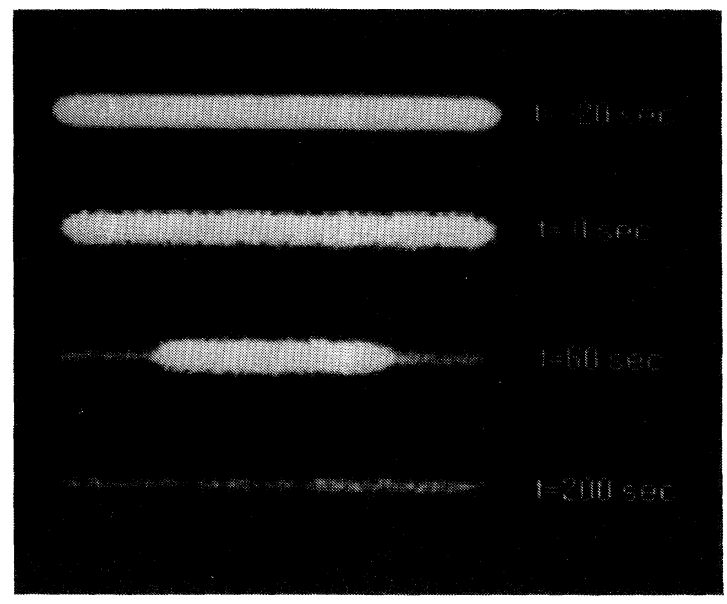

FIG. 3. Four representative images of the scattered intensity from the laser beam crossing the sample cell. In (a) the suspension is still sheared and the image appears as a brought light band, as the flow is stopped (b) single speckels are discernible. In (c) $t=60 \mathrm{~s}$ after the start of solidification the two phases are clearly distinguished by their difference in scattering intensity (high intensity $=$ melt; low $=$ solid). After complete solidification (d) the whole scattering volume again shows a homogeneous intensity but on the low level of the crystal.

sity is spatially homogeneous but on a much lower level.

The intensity parallel to the image of the laser beam is measured for some 100 pictures per crystallization run, taken at time intervals corresponding to approximately $10 \mu \mathrm{m}$ progress of the crystallization front. In Fig. 4 . we plot a selection of these. The boundary between crystal and fluid appears to be slightly blurred. This blurring is less pronounced for suspension parameters close to the phase boundary but may reach some $50 \mu \mathrm{m}$ for largest interaction. It is partly due to overshining effects but also gives evidence for a finite transition layer between crystal and fluid.

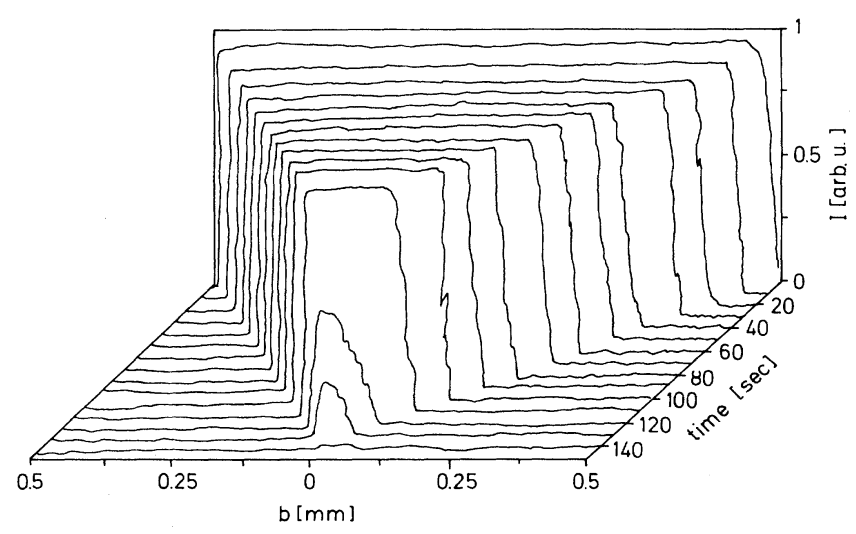

FIG. 4. The intensity distribution within the scattering volume plotted at different times after the start of solidification. Note that the time is plotted from back to front.

\section{WILSON-FRENKEL GROWTH}

These raw data are now evaluated for the growth velocities in the $\langle 110\rangle$ direction. The thickness of the two crystals is determined as the full width at half maximum of the intensity distributions shown in Fig. 5. It carries an absolute uncertainty of some $20 \mu \mathrm{m}$ but the error in relative thickness $x(t)$ needed to calculate the growth velocity is well below $1 \%$. We observe strictly linear growth that starts immediately after cessation of shear. This result is consistent with the findings of Aastuen et al. $[18,21]$ who observe linear growth after homogeneous nucleation.

The observation of layering induced heterogeneous nucleation followed by quasiepitaxial growth has been reported before [21]. We therefore concentrate here on the growth process itself and will report about detailed investigations of the nucleation scenario elsewhere. We note, however, that the bcc monolithic crystals nucleate with their (110) plane parallel to the cell wall, and therefore our growth direction perpendicular to the cell wall is $\langle 110\rangle$ as well. The measured interfacial velocities $v_{110}$ are shown in Fig. 6 as a function of the salt concentration $c$ at constant $\Phi_{A}=0.003$ and $\Phi_{B}=0.0022$. Note the strong influence of the increased surface group number in sample $B$, which overcompensates the larger volume fraction of sample $A$. Figure 7 shows $v_{110}$ as a function of $\Phi$ at zero added salt. Due to the large number of data on $x(t)$ the uncertainty concerning $v_{110}$ is well below $1 \%$. The slightly larger scatter of data points is due to the remaining uncertainties in $c$ (respectively, $\Phi$ ), which strongly influence the value of $v_{110}$. With increasing interaction the velocities vary over roughly four orders of magnitude in the vicinity of the phase transition and reach a plateau value of approximately $9 \mu \mathrm{m} \mathrm{s}^{-1}$ at the strongest interaction. In all three series the points given by the stars are measured in the regime of equilibrium coexistence. Within this region, denoted by filled circles in Fig. 1, the suspension only partially crystallizes, and two wall nucleated crystals surround a core of slightly less dense colloidal fluid.

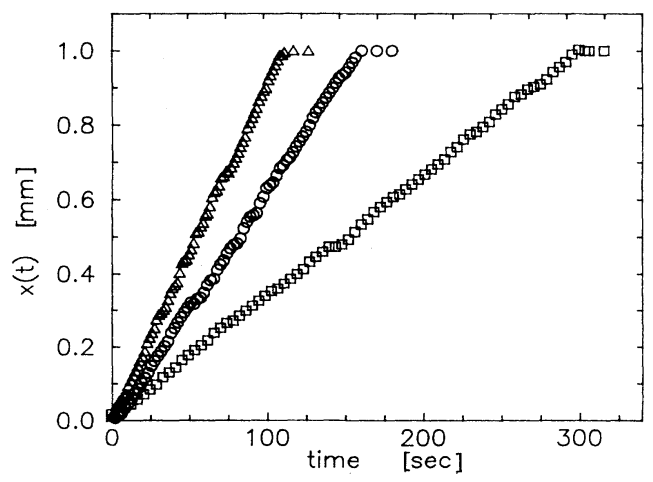

FIG. 5. Thickness $x(t)$ of the crystalline phase plotted as a function of time for different suspension parameters. The triangles are $B$ particles at $\Phi=0.0019$ and $c=0$; the circles are $A$ particles at $\Phi=0.003$ and $c=0.27 \mu \mathrm{mol}^{-1}$, and the squares are $B$ particles at $\Phi=0.0022$ and $c=1.08 \mu \mathrm{moll}^{-1}$. 


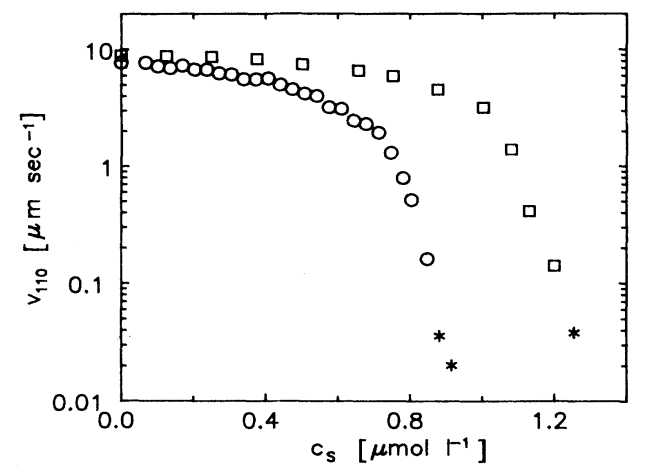

FIG. 6. The measured interfacial velocities $v_{110}$ as a function of salt concentration, the circles are data on the $A$ particles at the fixed volume fraction $\Phi_{A}=0.003$ and the squares are data on the $B$ particles at $\Phi_{B}=0.0022$. In both curves the stars denote points measured in the coexistence region.

Previous discussions of the radial velocities after homogeneous nucleation suffered from uncertainties concerning the particle concentrations, the position of the phase boundary and the condition of complete deionization which were considerably larger than in the present study. Our data may therefore be subjected to rigorous quantitative analysis, and one main interest will lie in the confirmation of the suggested Wilson-Frenkel growth law. The existence of a systematically measured high precision data set allows us to develop a quantitative measurement of the chemical potential difference between metastable melt and solid. Furthermore, it allows us to investigate the structure of the interfacial region.

The suggested growth dynamics of former studies approximated the difference in chemical potential as the reduced difference in particle number densities of metastable melt $\rho$ and of the fluid at melting $\rho_{m}$; $\Delta \mu \sim\left(\rho-\rho_{m}\right) / \rho_{m}$. This was appropriate, since only data on the density dependence of $v$ were available. However, changes in the screening parameters due to changes in density are then neglected. The interpretation of salt concentration or charge number dependent measure-

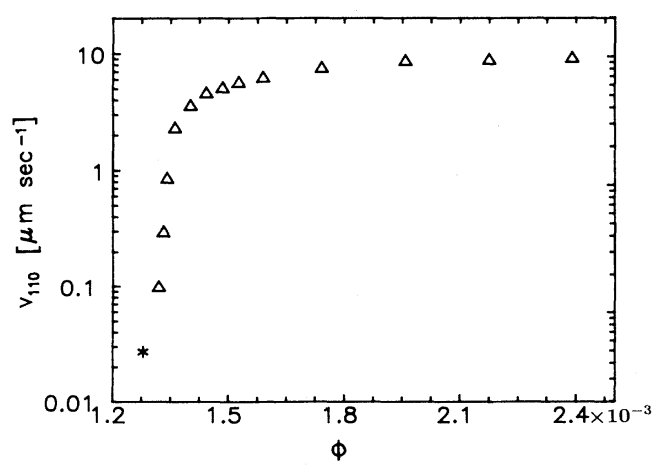

FIG. 7. The measured interfacial velocities $v_{110}$ as a function of the volume fraction $\Phi_{B}$ of $B$ particles at no added salt. The stars again denote points measured in the coexistence region. ments obviously is impossible. We therefore suggest to formulate $\Delta \mu$ in terms of a reduced energy density which is derived starting from the pair energy of electrostatic interaction between two particles separated by a distance $r$. We use the recently tested modified DLVO approximation (MDA) [10,12]:

$$
V(r)=\frac{Z_{\mathrm{PBC}}^{2} e^{2}}{4 \pi \epsilon \epsilon_{0}}\left[\frac{\exp (\kappa a)}{1+\kappa a}\right]^{2} \frac{\exp (-\kappa r)}{r},
$$

with the screening parameter $\kappa$ given by

$$
\kappa^{2}=\frac{e^{2}}{\epsilon \epsilon_{0} k_{B} T}\left(Z_{\mathrm{PBC}} \rho+2000 N_{A} c\right) .
$$

Here $Z_{\text {PBC }}$ is the effective or renormalized charge, calculated according to the procedures of Alexander et al. [9] under conditions of constant dissociation equilibrium [17]. For the present particles the effective charge numbers at $\Phi_{A}=0.003$ and $\Phi_{B}=0.0022$ and no added salt are $Z_{\mathrm{PBC}, A}=395$ and $Z_{\mathrm{PBC}, B}=450$, respectively. $\epsilon \epsilon_{0}$ is the dielectric permittivity of the suspension, $e$ is the elementary charge, $k_{B}$, the Boltzmann factor, $T$ the temperature, and $N_{A}$ Avogadro's number. We assume pairwise interaction of a central particle with an effective number $\alpha$ of neighbors and sum over all pairs in a given volume:

$$
\Pi=\alpha \frac{1}{2} V(r) \rho \text {. }
$$

We note that $\Pi$ formally has the dimension of an osmotic pressure. In the vicinity of the phase transition the chemical potential difference between melt and crystal is then approximated by the difference between melt and fluid at melting. This procedure is equivalent to the construction of a reduced undercooling or overpressure in atomic systems.

$$
\begin{aligned}
\Delta \mu & =B \Pi^{*} \\
& =B \frac{\Pi-\Pi_{m}}{\Pi_{m}} \\
& =B\left(\frac{\frac{\exp (-\kappa r)}{r} \rho-\frac{\exp \left(-\kappa_{m} r_{m}\right)}{r_{m}} \rho_{m}}{\frac{\exp \left(-\kappa_{m} r_{m}\right)}{r_{m}} \rho_{m}}\right) .
\end{aligned}
$$

Note that although the first two terms on the right hand side of Eq. (3) drop out of Eq. (5), the charge dependence is retained through different $\kappa$ for the two kinds of particles. Equation (5) neglects the contribution of entropic terms, which are assumed to be small compared to the respective potential differences. Very close to the phase boundary this may not necessarily be true. The new prefactor $B$ comprises changes in these entropic terms, in the effective number of neighbors, and in the effective charge numbers. All three, however, may easily be incorporated explicitly into the analysis. A more severe objection may be raised on theoretical grounds concerning the use of the melting line instead of the freezing line as point of reference. To a good first approximation this will produce a shift of the curves in a plot of $v_{110}$ versus $\Pi^{*}$ towards higher values of $\Pi^{*}$, but not qualitatively alter the re- 
sults. A detailed discussion of this point will be reported together with comprehensive data on growth at coexistence in a later publication.

We now will check our data for Wilson-Frenkel growth and our approximation of $\Delta \mu$ for consistency. In Fig. 8 we plot $v_{110}$ versus $\Pi^{*}$ for all three series. The data points coincide on a single curve within the experimental error. This provides strong evidence for the validity of our description in terms of reduced energy density $\Pi^{*}$. We qualitatively observe an initially linear increase in $v_{110}$ and a pronounced saturation for large $\Pi^{*}$. The four points at negative $\Pi^{*}$ correspond to the measurements at coexistence. They will not be included in further analysis since the prefactor in the Wilson-Frenkel law is predicted only to describe crystallization dynamics without phase separation and evolution of equilibrium density differences. In fact, dendritic growth was recently reported for this latter case [40].

We fit all data for positive $\Pi^{*}$ to a Wilson-Frenkel law with the limiting velocity $v_{\infty}$ and $B$ as the only free parameters. The best parameters of the fits to each single series are given in Table I. As was seen from Fig. 8, the values for $v$ coincide within the standard deviation of less than 3\%; respectively, the accumulated experimentally uncertainties which are below $5 \%$. The best fit to all data gives values of $v_{\infty}=9.1 \pm 0.1 \mu \mathrm{m} \mathrm{s}^{-1}$ for the limiting growth velocity and $B=6.7 \pm 0.1 k_{B} T$, which are reproducible within $1 \%$. This very satisfying agreement between experimental data and predicted behavior quantitatively confirms the suggested Wilson-Frenkel behavior for solidifying colloidal melts of highly charged latex spheres. From those points measured at coexistence one recognizes further that the simple description using a constant $v_{\infty}$ will not suffice to describe the crystallization behavior below melting where significant density differences have to develop.

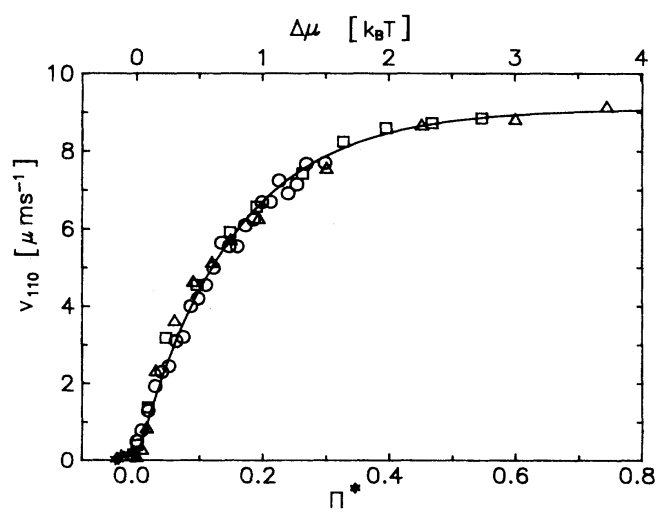

FIG. 8. The growth velocity $v_{110}$ plotted versus the reduced density difference $\Pi^{*}$ for all three experimental series (symbols as in Figs. 5 and 6). Within the experimental error all data points coincide on one master curve. A Wilson-Frenkel law [Eq. (1)] is fitted to the data with positive $\Pi^{*}$ and shown as solid line. The best fit parameters are $v_{\infty}=9.1 \pm 0.1 \mu \mathrm{m} \mathrm{s}^{-1}$ and $B=6.7 \pm 0.1 k_{B} T$. The chemical potential difference $\Delta \mu$ between solid and melt used in the upper $x$ axis was derived from $\Pi^{*}$ using the conversion factor $B$.
Furthermore, the good fit a posteriori verifies our formulation of the potential difference driving the phase transition. Therefore it is possible to interpret the fit parameter $B$ as a conversion factor between the reduced energy density and the chemical potential difference between melt and solid. This is used in the upper scale of Fig. 8. We note explicitly that the good agreement between all three series plotted versus $\Pi^{*}$ is somewhat unexpected, since the derivation of the energy density bases on rather crude estimates. Aastuen et al. [18] only used $\rho$ to estimate the conversion factor and reported a value larger by some $15 \%$, however, of much larger uncertainty. We further note that although our value has been determined with great precision it will be subject to further systematic changes, once entropic effects are incorporated into the derivation of $\Pi^{*}$. We estimated these corrections to be less than $10 \%$ for dilute systems. A value of $B=6.7 \pm 0.6$ therefore should facilitate a quick but reliable estimate of thermodynamic quantities useful, for example, in further investigations on crystallization dynamics.

\section{INTERFACIAL STRUCTURE}

We have shown that in highly charged systems growth is driven by the difference in chemical potential between melt and solid. At large $\Delta \mu$, however, it is limited by a kinetic process leading to a finite and constant maximum growth velocity. Thus we confirmed the characteristics of Wilson-Frenkel growth for our systems of highly charged particles. To gain further insight into the nature of this kinetic process we will take a closer look at the prefactor $v_{\infty}$ and consider different processes which in principle may determine the growth velocity. Even in our comparably simple isotherm and isochoric system we have to consider and discriminate transport towards the interface, relaxation into the crystalline structure, and the possible formation of transient, intermediate or precursor states.

Both in hard sphere systems and in charged systems at elevated volume fractions the evolution of a depletion layer around growing crystals has been observed $[25,26,40]$. This leads to growth control by collective transport across a significant density modulation and to nonlinear growth as a function of time. We cannot completely rule out the formation of depletion zones close to the interface but rather observe a transient homogeneous density decrease throughout the melt. In fact, preliminary measurements of the homogeneous nucleation rate as function of time show an order of magnitude decrease after roughly $20 \%$ of the suspension are solidified, indicating a transient decrease in the packing fraction of the melt by some $10 \%$. It applies to the complete molten region and is caused by the extremely high collective diffusion coefficient [14]. As can be seen from Fig. 6, however, far from the phase boundary, even a moderate reduction of the packing fraction will not considerably change the growth velocity. Moreover, we did not observe any deviation from strictly linear growth for measurements performed above melting. Therefore we conclude that even if such effects are present in our system 
they will not significantly influence the limiting velocity $v_{\infty}$.

In previous work on homogeneously nucleated crystals it was inferred from the round shape of the crystallites that their interface is rough, and no energy barrier is present to slow down the arrangement of particles into the crystal lattice. Such a behavior has also been observed in computer simulations of a growing planar 100 face of a face centered cubic crystal (fcc) [30]. There $v_{\infty}$ was equated with the thermal velocity of the LennardJones particles. In order to reproduce the saturation behavior observed in the present work, $v_{\infty}$ should depend on a transport coefficient which does not vary significantly over the whole investigated range of the phase diagram. It has been suggested to use the short time self-diffusion $D_{S}$ [18] which for dilute systems takes the Stokes-Einstein value $D_{S}=D_{0}=k_{B} T / 6 \pi \eta r$. Alternatively the long time self-diffusion coefficient which recently has been determined by forced Rayleigh scattering may be used. Its value stays close to the value at melting $D_{L} \sim 0.1 D_{S}$ throughout the metastable melt at the densities under investigation here $[36,41]$.

To quantify the time scales involved we consider a planar interface of thickness $L=d_{110}$, the lattice spacing in the $\langle 110\rangle$ direction. From the measured limiting velocity we derive a propagation time $\tau_{110}=d_{110} / v_{\infty}=55 \mathrm{~ms}$ during which the interface progresses by one crystal plane. The boundary of the interface is given on the crystalline side by the last layer of full crystalline order. The position at which the spatial correlations in $\langle 110\rangle$ direction have decayed is defined as the fluid side boundary. From the point of view of the crystal side the melt is homogeneous beyond the boundary. A particle situated here has a mean distance to its later target place in the crystal lattice of $0.4 d_{110}$, which simply is the average distance of points homogeneously distributed in a sphere of diameter $d_{110}$ to the sphere center. Using the mean squared displacement of a diffusing particle given as $\left\langle r^{2}\right\rangle=6 D t$ we estimate the average time $\tau_{D}$ for a particle to diffuse this distance as $\tau_{D}=0.4 d_{110}^{2} / 6 D_{0}=1.7 \mathrm{~ms}$, if $D_{0}$ and $\tau_{D}=16.7 \mathrm{~ms}$ and $D_{L}$ is used. In both cases $\tau_{D}$ is much smaller than $\tau_{110}$ and several of these time steps elapse before the interface has progressed by his own thickness, i.e., the particle has reached its target place. Setting the interfacial thickness to one layer spacing $L=d_{110}$ and using $D_{L}$ the maximum value for the limiting velocities becomes $v_{\infty}=33 \mu \mathrm{m} \mathrm{s}^{-1}$. Larger values for $L$ would lead to even larger velocities. It is interesting to note that using our analysis on the data of Aastuen et al. [nearest neighbor spacing $d=\left(2 \times 10^{18}\right)^{-1 / 3}=794 \mathrm{~nm}$ and $\left.D_{L}=4.38 \times 10^{-13} \mathrm{~m}^{2} \mathrm{~s}^{-1}[18]\right]$ predicts a limiting radial growth velocity of $v_{\infty, r}=21.7 \mu \mathrm{s}^{-1}$, which is in quantitative agreement with their observed value of $v_{\infty, r}=20$ $\mu \mathrm{s}^{-1}$. While this strongly supports the idea of no energy barrier being present in the growth of homogeneously nucleated crystals, the experimentally observed limiting velocity in our case of growth of a planar (110) crystal face is much smaller than the predicted one. We therefore have to investigate further on possible limiting processes.

In a more recent computer simulation [31] it was ob- served that under otherwise equal conditions the growth in the $\langle 111\rangle$ direction of a fcc Lenard-Jones crystal is much slower as compared to the previously investigated $\langle 100\rangle$ direction. Detailed analysis of the interfacial kinetics revealed that although both interfaces were some layers thick only on the (100) face the particles moved straight to their target places. On the (111) face first areas of in plane hexagonal order were formed which then registered on the underlying plane by means of an activated cooperative process. It was concluded that such a mechanism may be operative only on crystal faces with a choice of registering positions. The growth velocities as a function of undercooling showed a saturation behavior in a range where limitation by transport to the interface was not yet playing a significant role. At about half the melting temperature a pronounced decrease was visible due to the freezing of the activated process. In order to compare these results to our experiment we have to take the differences in crystal structure into account. No alternation in the stacking of layers is possible here. In the case of the bcc (110) face, however, two positions for registering are possible corresponding to the formation of two bcc twin forms. It seems very plausible that the observed slow growth velocities in our case are due to a similar mechanism as was observed in the case of the fcc (111) Lenard-Jones crystal face. In fact the monolithic crystals grown in the present experiment show a large amount of both lateral twin boundaries as well as an alternation of twins in the growth direction. These defects are not present in homogeneously nucleated crystallites growing from the bulk melt.

In a recent study [20] on the heterogeneous nucleation of charged spheres in a confined geometry, Grier and Murray showed a three step process with the formation of in plane fluid ordering proceeding the crystallization of this plane and the subsequent annealing. Both the solidification and the subsequent annealing were recorded in terms of the evolution of the sixfold bond orientational order parameter and the long time self-diffusion coefficient. It was observed that annealing was slower by roughly an order of magnitude. By assuming the annealing process to correspond to the above mentioned registering we estimate a registering-limited growth velocity of the interface as $v_{110}=3.3 \mathrm{~ms}^{-1}$ using $L=d_{110}$ and $D=0.01 D_{0}$. In turn the experimentally observed $v_{110}$ is consistent with an interfacial thickness of $L=9.1 / 3.3=2.7$ layers, which is close to the values observed in density functional calculations and in molecular dynamic simulations [33-35]. This would be experimental evidence of layering in colloidal melts adjacent to a growing crystal.

Finally, also a small amount of polydispersity may lead to a slowed growth in both scenarios via reactionlike processes. A single particle of different size present in a rough interface will lead to a local change of the driving thermodynamic force and simultaneously produce a packing fault which will influence further solidification. Within the scenario of a thick interface of registering layers such a particle will represent a pinning center preventing fast registration. Our particles are slightly 
more polydisperse than those used by Aastuen et al. [18]. Fits of the static structure factor give an upper bound of some $10 \%$ in size, which corresponds to a polydispersity of interaction potentials of approximately 3\% [37]. While the change in the interaction should be negligible in the derivation of a limiting velocity, it remains an open question whether the size polydispersity has a predominant influence on our data. A careful comparison between different growth directions for mixtures of differently sized particles should clarify this interesting question.

\section{CONCLUSION}

We have measured the growth velocities of planar (110) interfaces of colloidal bcc crystals with high accuracy and under systematic and precise variation of all relevant suspension parameters. In our measurements we focused on the investigation of growth under conditions which were not disturbed by simultaneous nucleation processes. The amount and the quality of the measured data facilitated a rigorous quantitative analysis, which yielded several important results.

Wilson-Frenkel growth could be unambiguously demonstrated for suspensions of highly charged colloidal spheres. Since these systems in some respect are the most simple realizations of crystallizing colloids and their parameters are accessible to well defined theoretical description, this provides a safe stepping stone for further investigations on more complicated systems. In particular, the study of growth in the presence of depletion and in the case of binary mixtures should be able to benefit from the present investigations.

The concept of a reduced density difference between melt and fluid at melting has proven suitable to unify the representation of all data series of the present study. Moreover, it was a posteriori justified to be a valid measure for the difference in chemical potential between the metastable melt and the equilibrium solid. The conversion factor $B=6.7 k_{B} T$ can be used to have a quick but reasonably accurate estimate of thermodynamic quantities from the experimental suspension data of the sample and of a single point along the melting line. Such a procedure may turn out to be extremely helpful in experiments where $\Delta \mu$ is needed from an independent source, for example, in the evaluation of nucleation rate densities in terms of classical nucleation theory.

Finally, the detailed discussion of the possible limiting processes responsible for the saturation behavior at large $\Delta \mu$ led to a refined understanding of the interfacial structure in the case of rough interfaces observed on roundish crystallites growing from the bulk melt as compared to the planar (110) interface of wall nucleated monolithic crystals. The differences observed between the growth mechanisms showed strong resemblance to those found in recent simulations of the (100) and (111) faces of fcc Lennard-Jones crystals. While not all open questions could be settled, we presented some evidence that in our case a registering of layers formed within a several layers thick interface is the predominant mechanism.

\section{ACKNOWLEDGMENTS}

The authors would like to thank H. Löwen, R. Klein, and the group of W. Dieterich for numerous critical discussions on diffusional processes and interfacial structures. Financial support by the Deutsche Forschungsgemeinschaft is also gratefully acknowledged.
[1] M. Volmer and A. Weber, Z. Phys. Chem. 119227 (1926); R. Becker and W. Döring, Ann. Phys. (Leipzig) 24, 719 (1935); D. Turnbull and J. C. Fischer, J. Chem. Phys. 17, 71 (1949); J. W. Christian, The Theory of Transformations in Metals and Alloys, 2nd ed. (Pergamon, Oxford, 1975), Chap. 10.

[2] H. A. Wilson, Philos. Mag. 50, 238 (1900).

[3] J. Frenkel, Phys. Z. Sowjetunion 1, 498 (1932).

[4] P. N. Pusey, in Liquids, freezing and the glass transition, Proceedings of the Les Houches Summer School, 1989, edited by J. P. Hansen, D. Levesque, and J. Zinn-Justin (Elsevier, Amsterdam, 1991), p. 637.

[5] Phase Transit. 21, (2-4) (1990), edited by B. J. Ackerson.

[6] B. J. Ackerson and N. A. Clark, Physica A 118, 221 (1983).

[7] T. Palberg, W. Härtl, U. Wittig, H. Versmold, and M. Würth, J. Phys. Chem. 96, 8081 (1992).

[8] T. Palberg, J. Kottal, F. Bitzer, R. Simon, M. Würth, and P. Leiderer, J. Coll. Interface Sci. 169, 85 (1995).

[9] S. Alexander, P. M. Chaikin, P. Grant, G. J. Morales, P. Pincus, and D. Hone, J. Chem. Phys. 80, 5776 (1984).

[10] F. Bitzer, T. Palberg, H. Löwen, R. Simon, and P. Leiderer, Phys. Rev. E 50, 2821 (1994).

[11] M. O. Robbins, K. Kremer, and G. S. Grest, J. Chem. Phys. 88, 3286 (1988).
[12] P. M. Chaikin, J. M. Di Meglio, W. Dozier, H. M. Lindsay, and D. W. Weitz, in Physics of complex and supramolecular fluids, edited by S. A. Safran and N. A. Clark (Wiley, New York, 1987), p. 65.

[13] R. Klein, in Structure and dynamics of strongly interacting colloids and supramolecular aggregates in solution, Vol. 396 of NATO Advanced Study Institut, Series C: Topic, edited by P. Tartaglia (Plenum, New York, 1991), p. 39.

[14] U. Genz and R. Klein, Physica A 171, 26 (1991).

[15] P. N. Pusey and W. van Megen, Nature 320, 340 (1989).

[16] L. P. Voegtli and C. F. Zukoski, J. Coll. Interface Sci. 141, 79 (1991).

[17] T. Palberg, W. Mönch, F. Bitzer, R. Piazza, and T. Bellini, Phys. Rev. Lett. 74, 4555 (1995).

[18] D. J. W. Aastuen, N. A. Clark, L. K. Cotter, and B. J. Ackerson, Phys. Rev. Lett. 57, 1733 (1986).

[19] I. S. Sogami and T. Yoshiyama, Phase Trans. 21, 171 (1990).

[20] D. G. Grier and C. A. Murray, J. Chem. Phys. 100, 9088 (1994).

[21] D. J. W. Aastuen, N. A. Clark, J. C. Swindal, and C. D. Muzny, Phys. Trans. 21, 139 (1990).

[22] W. Härtel, R. Klemp, and H. Versmold, Phase Trans. 21, 229 (1990)

[23] T. Okubo, J. Chem. Phys. 87, 3022 (1987). 
[24] K. E. Davis and W. B. Russel, Adv. Ceram. 21, 573 (1987).

[25] K. Schätzel and B. J. Ackerson, Phys. Rev. Lett 68, 337 (1992).

[26] K. Schätzel and B. J. Ackerson, J. Phys. Rev. E 483766 (1993).

[27] J. K. G. Dhont, C. Smits, and H. N. W. Lekkerkerker, J. Coll. Interface Sci. 152, 386 (1992).

[28] T. Palberg, M. Würth, J. Schwarz, and P. Leiderer, Prog. Colloid Polym. Sci. 98, 6 (1995).

[29] W. B. Russel, Phase Trans. 21, 127 (1990).

[30] J. Q. Broughton, G. H. Gilmer, and K. A. Jackson, Phys. Rev. Lett. 49, 1496 (1982).

[31] F. Burke, J. Q. Broughton, and G. H. Gilmer, J. Chem. Phys. 89, 1030 (1988).

[32] W. van Megen and S. Underwood, J. Phys. Condens. Matter 6, A181 (1994).
[33] H. Löwen, Phys. Rep. 237, 249 (1994).

[34] D. W. Marr and A. P. Gast, Phys. Rev. E 47, 1212 (1992).

[35] M. Nieswand, A. Majerhofer, and W. Dieterich, Phys. Rev. E 48, 2521 (1993).

[36] T. Palberg, M. Würth, R. Simon, and P. Leiderer; Prog. Colloid Polym. Sci. 96, 62 (1994).

[37] R. Krause, B. D'Aguanno, J. M. Mendez-Alcaraz, G. Nägele, R. Klein, and R. Weber, J. Phys. (Paris) Coloq. 3, C3-4459 (1991).

[38] E. B. Sirota, H. D. Ou-Yang, S. K. Sinha, P. M. Chaikin, J. D. Axe, and Y. Fujii, Phys. Rev. Lett. 62, 1524 (1989).

[39] W. D. Dozier, H. M. Lindsay, and P. M. Chaikin, J. Phys. (Paris) Colloq. 46, C3-257 (1985).

[40] A. P. Gast and Y. Monovoukas, Nature 351, 533 (1991).

[41] H. Löwen, T. Palberg, and R. Simon, Phys. Rev. Lett. 70, 1557 (1993). 


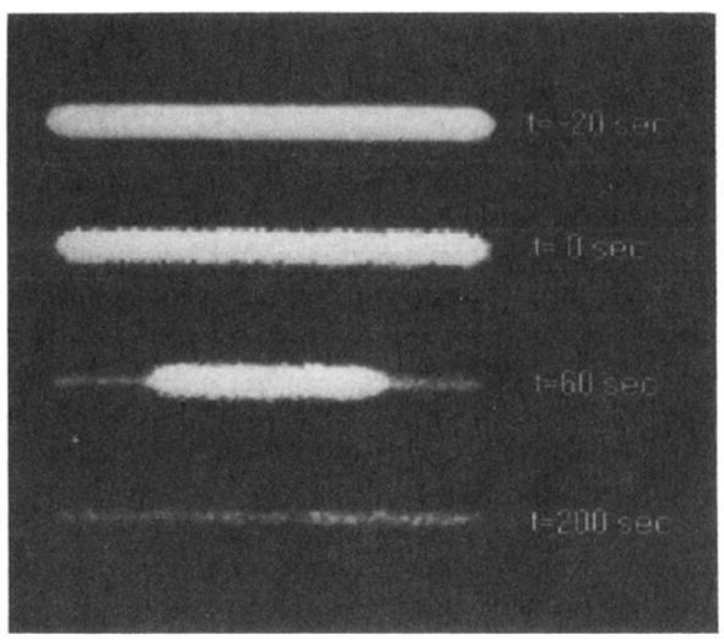

FIG. 3. Four representative images of the scattered intensity from the laser beam crossing the sample cell. In (a) the suspension is still sheared and the image appears as a brought light band, as the flow is stopped (b) single speckels are discernible. In (c) $t=60 \mathrm{~s}$ after the start of solidification the two phases are clearly distinguished by their difference in scattering intensity (high intensity $=$ melt; low $=$ solid). After complete solidification (d) the whole scattering volume again shows a homogeneous intensity but on the low level of the crystal. 